\title{
The Role of Secondary Electron Emission in the Charging of Thin-Film Phase Plates
}

\author{
M. Dries ${ }^{1}$, R. Janzen ${ }^{1}$, T. Schulze ${ }^{1}$, J. Schundelmeier ${ }^{1}$, S. Hettler ${ }^{1}$, U. Golla-Schindler ${ }^{2}$, B. Jaud ${ }^{2}$, \\ U. Kaiser ${ }^{2}$ and D. Gerthsen ${ }^{1}$ \\ ${ }^{1 .}$ Laboratory for Electron Microscopy, Karlsruhe Institute of Technology, Karlsruhe, Germany \\ ${ }^{2 .}$ Electron Microscopy Group of Material Science, University of Ulm, Ulm, Germany
}

In the past few years, physical phase plates (PP) have become a viable tool to enhance the contrast of weak-phase objects in transmission electron microscopy (TEM). Thin-film PPs, such as the Zernike and Hilbert PP, are based on the mean inner potential of microstructured thin films [1,2]. Typically, a thin amorphous carbon (aC)-film is applied, whose thickness is adjusted to induce a well-defined phase shift between unscattered and scattered electrons. However, the illumination with high-energy electrons initiates an irreversible degeneration of the aC-film, which causes electrostatic charging and affects the phase-shifting properties. Taking even advantage of charging, hole-free PPs were recently developed $[3,4]$.

Electrostatic charging plays a central role in the application of thin-film PPs. However, the mechanisms of charging are not well-understood. This work shows that charging is dominated by secondary electron emission. For this purpose, Hilbert PPs were fabricated from different materials to study their charging behavior under electron beam illumination. Besides aC-films, thin films of the metallic glass alloy $\mathrm{Pd}_{77.5} \mathrm{Cu}_{6.0} \mathrm{Si}_{16.5}$ (PCS) were used for PP fabrication. The PCS-alloy is characterized by an amorphous structure and a high electrical conductivity, which is three orders of magnitude higher than that of aC [5,6]. Moreover, the PCS-alloy exhibits a strong resistance towards oxidation, which suggests less charging of PCS-films.

Thin films of aC and the PCS-alloy were fabricated by electron beam evaporation and sputter deposition, respectively. Using a focused-ion-beam system, rectangular windows were structured into the thin films. The microstructured thin films were implemented in the back focal plane of a Philips CM200 FEG/ST transmission electron microscope. For use as a Hilbert PP, the film thickness has to be adjusted to a phase shift of $\pi$. At $200 \mathrm{keV}$ electron energy, this corresponds to a film thickness of $49 \mathrm{~nm}$ for aC and $19 \mathrm{~nm}$ for the PCS-alloy.

Fig. 1 shows phase-contrast TEM images and corresponding power spectra of an aC test object, which were acquired using an aC (Fig. 1a) or a PCS (Fig. 1b) Hilbert PP. The cut-on frequency is given by the distance between the PP edge and the zero-order beam and is marked by vertical white lines. The complementary behavior of the Thon-rings below and above the cut-on frequency demonstrates the desired phase-shifting properties. By choosing a relatively large cut-on frequency, obvious charging is avoided for the $\mathrm{aC}$ Hilbert PP in Fig. 1a. Despite the excellent electrical properties of the PCS-alloy, charging occurs for the PCS Hilbert PP as can be deduced from the strong distortion of the Thon-ring system in Fig. 1b. However, charging is significantly reduced if the PCS Hilbert PP is coated with a thin aC-layer as shown in Fig. 1c. Fig. 1 suggests that less charging occurs if aC is applied. It is noted, that for smaller cut-on frequencies (i.e. smaller distances between the PP edge and the zero-order beam) charging also occurs for the $\mathrm{aC}$ (Fig. 1a) and the aC/PCS (Fig. 1c) Hilbert PP. However, the stability under electron beam illumination is improved for the aC/PCS Hilbert PP, for which the carbon content is drastically reduced with respect to pure aC-films. 
Reduced charging in Figs. 1a,c is attributed to the low secondary electron emission of aC compared to the PCS-alloy. The emission of secondary and backscattered electrons was studied in a Zeiss NVision40 scanning electron microscope. The Hilbert PPs were mounted on a specific device, where the absorbed and the transmitted current can be measured separately with a potentiostat (BioLogic SP-200) and the specimen current monitor of the scanning electron microscope. The absorbed current to ground $\mathrm{I}_{S}$ was measured for different primary electron energies $\mathrm{E}$ from $0.5 \mathrm{keV}$ to $20 \mathrm{keV}$. Fig. 2 shows the absorbed current normalized with respect to the current of the incident electron beam $\mathrm{I}_{\mathrm{PE}}$ for $\mathrm{aC}$ and the PCSalloy. Positive values of $\mathrm{I}_{\mathrm{S}} / \mathrm{I}_{\mathrm{PE}}$ indicate positive charging and vice versa. Between $1 \mathrm{keV}$ and $3 \mathrm{keV}$ aC is negatively charged. Above $3 \mathrm{keV}$ the aC-film shows a small tendency for charging. Although the blue curve is close to zero, $\mathrm{I}_{\mathrm{S}} / \mathrm{I}_{\mathrm{PE}}$ takes finite values, which are below the measurement accuracy. The PCSalloy has a negative charge below $5 \mathrm{keV}$, but is positively charged for electron energies up to $20 \mathrm{keV}$. The low tendency for charging of $\mathrm{aC}$ and the positive charging of the PCS-alloy are consistent with the observations in Fig. 1. It is noted, that electron energies are much higher in TEM. However, the curves in Fig. 2 show trends, which might continue towards higher energies.

The observation of charging implies poor electrical conductivity. Otherwise, any positive or negative charge balance would be compensated by an electrical current to ground. We assume that our Hilbert PPs are well-grounded and that $\mathrm{aC}$ as well as the PCS-alloy have a sufficient electrical conductivity for the low beam currents in TEM. One possible explanation for charging is beam-induced contamination. Low energy secondary electrons, which get trapped in the electrically insulating contamination layer, could then cause the formation of an electrical dipole layer at the interface between the contamination and the PP film.

[1] R. Danev and K. Nagayama, Ultramicroscopy 88 (2001), p. 243.

[2] R. Danev and K. Nagayama, J. Phys. Soc. Jpn. 73 (2004), p. 2718.

[3] M. Malac et al., Ultramicroscopy 118 (2012), p. 77.

[4] R. Danev et al., PNAS 111 (2014), p. 15635.

[5] B. Chelluri and R. Kirchheim, J. Non-Cryst. Solids 54 (1983), p. 107.

[6] M. Morgan, Thin Solid Films 7 (1971), p. 313.

[7] Financial support by the Deutsche Forschungsgemeinschaft (DFG).
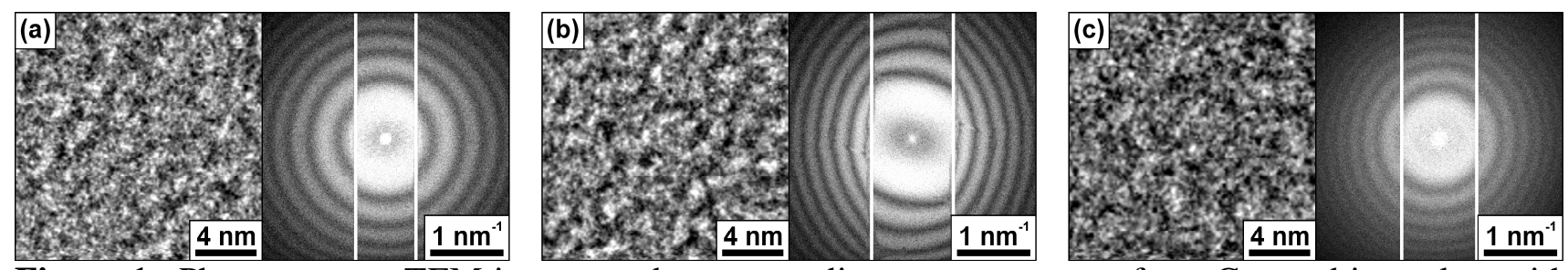

Figure 1. Phase-contrast TEM images and corresponding power spectra of an aC test object taken with (a) an aC Hilbert PP, (b) a PCS Hilbert PP and (c) an aC/PCS Hilbert PP at $200 \mathrm{keV}$.

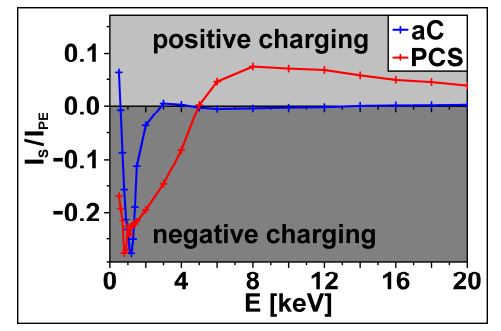

Figure 2. Normalized specimen current as a function of the primary electron energy $\mathrm{E}$ for $\mathrm{aC}$ and the PCS-alloy ( $\mathrm{I}_{\mathrm{S}}$ : Absorbed current to ground, $\mathrm{I}_{\mathrm{PE}}$ : Current of the incident electron beam). 